\title{
The first binary pulsars and what they told us about binary evolution
}

\author{
Dipankar Bhattacharya ${ }^{1}$ \\ ${ }^{1}$ Inter-University Centre for Astronomy and Astrophysics \\ Post Box 4, Ganeshkhind, Pune 411007, India \\ email: dipankar@iucaa.in
}

\begin{abstract}
The first few binary pulsars revealed the richness of evolution possible in binary systems containing neutron stars. Products of different evolutionary routes, in high and low mass binaries, as well as examples of evolution affected by the pulsar wind were among the first ten objects discovered. This article presents a historical review of the impact of binary pulsars on the early development of ideas regarding the evolution of neutron stars in binary systems.
\end{abstract}

\section{Introduction}

Neutron stars in binary systems were first recognised as being members of bright, accreting X-ray binary sources [Zeldovich \& Guseynov (1965)]. Several years of intense work on the evolution of such objects had followed when the first binary pulsar, PSR B1913+16, was discovered [Taylor \& Hulse (1974); Hulse \& Taylor (1975)]. This remarkable double neutron star system was immediately recognised as the product of Xray binary evolution, and its properties revealed new aspects of the late stages of binary evolution. Lessons learnt from the first few binary pulsars discovered in the next decade and a half helped develop the current picture of the evolution of neutron stars in binary systems. In this article I will discuss this development, based on the first ten pulsars of this class listed in Table 1 . The list includes nine pulsars with binary companions and the first millisecond pulsar PSR B1937+21, which though currently isolated, bears all signs of having been processed in a binary.

Table 1. The first ten binary and millisecond pulsars, in order of their discovery

\begin{tabular}{l|c|c|c|c|c|c|}
\hline Pulsar & $P_{\text {spin }}(\mathrm{s})$ & $\log B_{\text {surf }}(\mathrm{G})$ & $P_{\text {orb }}(\mathrm{d})$ & eccentricity & $M_{\text {comp }}\left(M_{\odot}\right)$ & ref. \\
\hline B1913+16 & 0.059 & 10.358 & 0.323 & 0.617 & 1.44 & 1 \\
B0655+64 & 0.196 & 10.068 & 1.029 & $7.5 \times 10^{-6}$ & $\sim 0.8$ & 2 \\
B0820+02 & 0.865 & 11.483 & 1232.404 & 0.012 & $\sim 0.5$ & 3 \\
B1937+21 & 0.00156 & 8.612 & - & - & - & 4 \\
B1953+29 & 0.00613 & 8.635 & 117.349 & $3.3 \times 10^{-4}$ & $\sim 0.2$ & 5 \\
B1831-00 & 0.521 & 10.874 & 1.811 & 0.0045 & $\sim 0.07$ & 6 \\
B2303+46 & 1.066 & 11.896 & 12.339 & 0.658 & $\sim 1.4$ & 6 \\
B1820-11 & 0.280 & 11.799 & 357.762 & 0.795 & $\sim 0.8$ & 7 \\
B1855+09 & 0.00536 & 8.496 & 12.327 & $2.2 \times 10^{-5}$ & 0.27 & 8 \\
B1957+20 & 0.0161 & 8.223 & 0.382 & $\sim 0.0$ & $\sim 0.025$ & 9 \\
\hline
\end{tabular}

Notes:

The surface magnetic field $B_{\mathrm{surf}}$ above is the dipole component estimated from spin period and spin-down rate. Companion masses $M_{\mathrm{comp}}$ are derived from relativistic orbital parameters for $\mathrm{B} 1913+16$ and $\mathrm{B} 1855+09$, while in other cases they are estimates based on the mass function, assuming a pulsar mass of $1.4 M_{\odot}$ and an inclination of $60^{\circ}$. For B $0820+02$ the mass is constrained by optical observations.

Discovery references:

1. Hulse \& Taylor (1975), 2. Damashek, Taylor \& Hulse (1978), 3. Manchester et al.(1978), 4. Backer et al.(1982), 5. Boriakoff et al.(1983), 6. Dewey et al.(1985), 7. Clifton \& Lyne (1986), 8. Segelstein et al.(1986) 9. Fruchter et al.(1988) 
From the first binary pulsar system B1913+16 alone, several important conclusions could be drawn, namely:

- A combination of low magnetic field strength and fast spin suggested spin-up of the neutron star by accretion [Smarr \& Blandford (1976)].

- The small orbital separation suggested that the binary system went through a common-envelope evolution [Smarr \& Blandford (1976)].

- A bound orbit of two neutron stars implied that the binary system remained bound after two supernovae. This suggested that neutron stars may receive substantial kicks at birth [Flannery \& van den Heuvel (1975)].

- Orbital shrinkage due to gravitational radiation was measured, highlighting the importance of gravitational radiation in the evolution of compact star binary systems [Weisberg \& Taylor (1981)].

Lessons derived from subsequent discoveries listed in Table 1 include:

- The existence of a new class of pulsars with low-mass binary companions

- Diverse evolutionary pathways of low-mass donor systems

- Reinforcement of the spin-up scenario

- Irradiation and evaporation of the companion of a pulsar

- Evolution of the magnetic fields of neutron stars

In the following sections I will briefly describe some of the issues mentioned above. Much of the discussion presented here may be found elaborated in the review article Bhattacharya \& van den Heuvel (1991).

\section{Spin-up by accretion}

Even before the discovery of the first binary pulsar, Davidson \& Ostriker (1973) had discussed in detail the idea of neutron star spin being affected by accretion of matter in a binary system. The inflowing matter forms an accretion disc, which is truncated at an inner radius, called the "Alfvén radius", determined by the magnetic field strength of the neutron star. Below this radius the accreting plasma is strongly coupled to the magnetic field and co-rotates with the neutron star. A spin equilibrium is achieved if the keplerian frequency at the Alfvén radius equals the neutron star's spin rate. If the neutron star is spinning slower than that, then the accreting matter would tend to spin it up, and vice versa. The lower the magnetic field, the closer to the neutron star the Alfvén radius will be, and the shorter will be the corresponding equilibrium spin period.

PSR B1913+16 distinguished itself from the rest of the known pulsar population at that time by its relatively low magnetic field strength (about two orders of magnitude below the average pulsar field), and a fairly fast spin period of $59 \mathrm{~ms}$. Smarr \& Blandford (1976) concluded that this combination implies that the neutron star must have experienced accretion in the binary system and has thereby been spun-up. The lower than average field strength allowed the spin-up process to achieve the observed short period.

For a given magnetic field strength $B$, the equilibrium period achieved in the spin-up process depends on the mass accretion rate $\dot{M}$ - the higher the mass inflow rate, the smaller is the Alfvén radius. For a dipole magnetic geometry the equilibrium spin period has the dependence $P_{\mathrm{eq}} \propto\left(B^{2} / \dot{M}\right)^{3 / 7}$. It was pointed out by Srinivasan \& van den Heuvel (1982) that this implies a lower limit to the period to which a neutron star of a given field strength could be spun up, corresponding to the maximum accretion rate, namely the Eddington rate $\dot{M}_{\mathrm{Edd}} \sim 10^{-8} M_{\odot} /$ yr. This relation between $P_{\text {eq }}$ and $B$, keeping $\dot{M}$ fixed at $\dot{M}_{\mathrm{Edd}}$ was named the "Critical spin-up line", and is referred to as simply the "Spin-up line" in current literature. 
In a paper presented at the 1981 Asia Pacific Regional Meeting in Bandung, Indonesia, Radhakrishnan \& Srinivasan (1984) argued that a whole population of spun-up pulsars must exist, and in a plot of pulsar magnetic field vs spin period, such objects would be found in the region bordered by the spin-up line on the left and the "Death Line" on the right. They called this population "Recycled Pulsars", a term still in current use. Subsequent discoveries of binary pulsars have amply borne out this prediction.

The discovery of the millisecond pulsar PSR B1937+21 [Backer et al.(1982)] put a spotlight on the recycling scenario. Here was a pulsar with a very short period and very low field, for which recycling would be a perfect explanation [Radhakrishnan \& Srinivasan (1982); Alpar et al.(1982)], but the lack of a companion was a problem. However, soon afterwards similar pulsars with binary companions were found [Boriakoff et al.(1983); Segelstein et al.(1986)], confirming the spin-up origin for millisecond pulsars.

\section{Orbital Evolution - mass transfer, spiral-in, supernova explosion}

With two neutron stars in the system, it was recognised that PSR B1913+16 must have descended from a high-mass X-ray binary system, in which the massive progenitor of the second born neutron star transferred mass onto the recycled pulsar. The orbital separation of the final system (periastron distance $\sim 1 R_{\odot}$ ) is however too small to fit the mass donating progenitor. Rapid shrinkage of orbit must therefore have taken place in the final stages of mass transfer. This implied highly non-conservative evolution of the binary in the late stages, via either a spiral-in process in a common envelope [Smarr \& Blandford (1976)] or a major outflow driven by super-Eddington mass transfer [Flannery \& van den Heuvel (1975)]. This sparked detailed radiation-hydrodynamic treatment of common envelope evolution, beginning with Bodenheimer \& Taam (1984) and continuing till today. In some cases common envelope evolution may lead to a complete merger of the binary components, leaving an isolated recycled pulsar [Bhattacharya \& van den Heuvel (1991); van den Heuvel \& Bonsema (1984)], but clear observational signature of this is difficult to find.

The birth of the second neutron star in PSR B1913+16 system would have been accompanied by a supernova explosion. It was known that the loss of more than half the total mass of the system in an explosion would unbind the binary. In this case, while a large eccentricity has been imparted, the system has however remained bound. Flannery \& van den Heuvel (1975) suggested that a slight asymmetry in the supernova could impart on the resulting neutron star a substantial kick (several hundred $\mathrm{km} / \mathrm{s}$ ) and in some cases this kick may be suitably directed to counteract the effect of sudden mass loss, thus keeping the system bound. Subsequently, this concept has been widely applied in modelling the formation of diverse types of binary systems containing neutron stars.

\section{Different classes of binary pulsars}

With the first few binary pulsar discoveries, it became clear that the population consists of two major classes. Objects like PSR B1913+16 and PSR B0655+64 descend from high mass binaries in which the donor star is heavier than the neutron star. Spiral-in evolution in the common envelope phase leaves a very tight final orbit in these cases. If there is a second supernova in the system, then an eccentric binary with two neutron stars is formed as in B1913+16 system (or the binary is disrupted). If the secondary avoids a supernova explosion, then a heavy white dwarf companion in a near circular orbit, as in B0655+64, would result. Such objects are called High Mass Binary Pulsars (HMBP). PSR B2303+46, a pulsar in an eccentric orbit with a heavy white dwarf companion, 
joins this class of binary systems, but the pulsar here was formed after the white dwarf and is not recycled. Another non-recycled pulsar in Table 1 is B1820-11, which has an unevolved main-sequence companion.

A second class of binary systems gives rise to objects like B0820+02, B1953+29, B1831$00, \mathrm{~B} 1855+09$ or B1957+20. Here the companion is a low mass white dwarf that can only form due to mass transfer in a binary system. The orbits are nearly circular and span a wide range periods - from hours to years. These Low Mass Binary Pulsars (LMBP) typically descend from Low Mass X-ray Binary (LMXB) systems where the mass donor is lighter than the neutron star. Mass transfer tends to widen such binaries, and hence very wide orbits, such as in B0820+02, become possible. To sustain mass transfer in a widening orbit, the radius of the donor star must grow, which it does by nuclear evolution in the stellar core if the donor makes the first contact in the subgiant or the giant branch. An inert, degenerate core grows within the donor as the nuclear evolution proceeds, and its radius increases correspondingly, until the envelope mass is drained leaving only the core as a low-mass white dwarf remnant [Joss \& Rappaport (1983); Webbink, Rappaport \& Savonije (1983)]. The larger the mass of the core, the larger would have been the radius, so this branch of evolution predicts a clear correlation between the remnant mass and the final orbital period of the binary [Joss, Rappaport \& Lewis (1987)]. The stellar evolution scenario could thus be tested by measuring the orbital periods and white dwarf masses in such LMBP systems. Accurate measurement of the white dwarf mass in these systems have proved difficult, however. The first observational confirmation of this relation therefore had to wait several years [van Kerkwijk et al.(2004)]. Another correlation was predicted between the orbital period and the eccentricity of such systems [Phinney (1992)] - tidal interaction with the convective envelope of the donor being responsible for pumping the eccentricity of the binary system. Observations have been found to broadly agree with the predicted correlation [Phinney \& Kulkarni (1994)].

The requirement that the first contact be made while the donor star is evolved restricts this branch of evolution to systems that are relatively wide - orbital periods starting with a day or more and then lengthening by an order of magnitude in the course of evolution. If the initial binary is much tighter, then a different evolutionary course is followed. Angular momentum loss by gravitational radiation and magnetic stellar wind become the primary drivers of mass transfer in such systems, whose orbits consequently shrink and can reach orbital periods of tens of minutes [Rappaport, Verbunt \& Joss (1983);Webbink, Rappaport \& Savonije (1983)]. The compact LMBP B1957+20 is a likely product of such an evolution.

A further twist to the evolutionary path of compact LMBPs - that of interaction of pulsar wind with its companion - was surmised, and then witnessed. The isolated nature of the first millisecond pulsar B1937+21, while bearing all characteristics of a recycled pulsar, gave rise to the speculation that the companion may have been evaporated by intercepting the radiation and particle wind of the pulsar. X-ray heating of the donor in a compact LMXB may drive a wind and a high rate of mass transfer, spinning the neutron star up to a very short period, until a very light $\left(\sim 10^{-2} M_{\odot}\right)$ secondary is left [Ruderman, Shaham, Tavani \& Eichler (1989)]. At this point, mass transfer rate would drop rapidly, allowing the neutron star to turn on as a pulsar, producing radiation and particle wind that would then ablate its companion [Ruderman, Shaham \& Tavani (1989)]. While these theoretical scenarios were in the process of publication, PSR B1957+20 was discovered, in which the pulsar is indeed driving a strong wind from its very low mass companion. 
Such pulsars have subsequently been christened "Black Widows", of which many more examples continue to be found.

\section{Magnetic field evolution}

One of the key factors determining the extent of spin-up of a neutron star is its magnetic field strength. Recycled pulsars, in general, are found to have short spin periods because their field strengths are on an average considerably lower than that of the isolated pulsar population. In the early days, the lower field strengths of the recycled neutron stars were attributed to their age: these neutron stars are older because they have had to wait for their companion stars to evolve and transfer mass. The prevalent view at that time was that the magnetic field of all neutron stars decay in the time scale of a few million years due to ohmic dissipation [Gunn \& Ostriker (1970)]. Detailed studies of binary pulsars, however, radically altered this view.

With the discovery of several millisecond pulsars it was realised that the observed population was not sustainable if their magnetic fields continued to decay after recycling. The idea of a non-decaying "asymptotic field" at $\sim 10^{8} \mathrm{G}$ was introduced [Bhattacharya \& Srinivasan (1986); van den Heuvel, van Paradjis \& Taam (1986)]. Some of the white dwarf companions in binary pulsar systems could be studied optically, and their cooling age determined. This led to the conclusion that the magnetic field of all these recycled pulsars were also long-lived, so the field decay could level off at values ranging all the way from $\sim 10^{8} \mathrm{G}$ to $\sim 10^{12} \mathrm{G}$ [Kulkarni (1986)]. Synthesising available information from different sources, including binary pulsars and the kinematic properties of isolated pulsars, Bailes (1989) concluded that the magnetic field decay exhibited by radio pulsars is a direct consequence of binary evolution. Over time, this has become the accepted picture of the evolution of pulsar magnetic fields.

\section{Conclusions}

The discovery of binary pulsars provided an excellent opportunity to test and augment ideas of binary evolution developed in the context of X-ray binary systems. New evolutionary pathways came to light, including those influenced by irradiation of the binary companion of the neutron star. Study of binary pulsars also radically changed our ideas of the magnetic field evolution in neutron stars. Today over 400 binary and millisecond pulsars are known. Foundations of the evolutionary scenarios describing them were laid already with the first ten such objects discovered. Later observations have continued to develop on these ideas and filled in several missing links.

\section{Acknowledgements}

I thank the organisers for the invitation to participate in this historic IAU symposium and for the excellent hospitality at Jodrell Bank.

\section{References}

Alpar, M. A., Cheng, A. F., Ruderman, M. A., \& Shaham, J. 1982, Nature, 300, 728

Backer, D. C., Kulkarni, S. R., Heiles, C., Davis, M. M., \& Goss, W. M. 1982, Nature, 300, 615

Baliles, M. 1989, ApJ, 342, 917

Bhattacharya, D. \& Srinivasan, G. 1986, Current Science, 55, 327

Bhattacharya, D. \& van den Heuvel, E. P. J. 1991, Physics Reports, 203, 1 
Bodenheimer, P. \& Taam, R. E. 1984, ApJ, 280, 771

Boriakoff, V., Buccheri, R., \& Fauci, F. 1983, Nature, 304, 417

Clifton, T. R. \& Lyne A. G. 1986, Nature, 320, 43

Damashek, M., Taylor, J. H., \& Hulse, R. A. 1978, ApJ, 225, L31

Davidson, K. \& Ostriker, J. P. 1973, ApJ, 179, 585

Dewey, R. J., Taylor, J. H., Weisberg, J. M., \& Stokes, G. H. 1985, ApJ, 294, L25

Flannery, B. P. \& van den Heuvel, E. P. J. 1975, A\& A, 39, 61

Fruchter, A. S., Stinebring, D. R., \& Taylor, J. H. 1988, Nature, 333, 237

Gunn, J. E. \& Ostriker, J. P. 1970, ApJ, 160, 979

Hulse, R. A. \& Taylor, J. H. 1975, ApJ, 195, L51

Joss, P. C. \& Rappaport, S. A. 1983, Nature, 304, 419

Joss, P. C., Rappaport, S., \& Lewis, W. 1987, ApJ, 319, 180

Kulkarni, S. R. 1986, ApJ, 306, L85

Manchester, R. N., Lyne, A. G., Taylor, J. H., Durdin, J. M., Large, M. I., \& Little, A. G 1978, MNRAS, 185, 409

Phinney, S. 1992, Philosophical Transactions: Physical Sciences and Engineering, 341, 39

Phinney, E. S. \& Kulkarni, S. R. 1994, ARAA, 32, 591

Radhakrishnan, V., \& Srinivasan, G., 1982, Current Science, 51, 596

Radhakrishnan, V., \& Srinivasan, G., 1984, in: Proceedings of the Asia Pacific Regional Meeting in Astronomy, held in Banding, Indonesia, August 24-29, 1981, eds. B. Hidayat \& M.W. Feast, p. 423

Rappaport, S., Verbunt, F., \& Joss, P. C. 1983, ApJ, 275, 713

Ruderman, M., Shaham, J., \& Tavani, M. 1989, ApJ, 336, 507

Ruderman, M., Shaham, J., Tavani, M., \& Eichler, D. 1989, ApJ, 343, 292

Segelstein, D. J., Rawley, L. A., Stinebring, D. R., Fruchter, A. S., \& Taylor, J. H. 1986, Nature, 322,714

Smarr, L. L. \& Blandford, R. D. 1976, ApJ, 207, 574

Srinivasan, G. \& van den Heuvel E. P. J. 1982, A\&A A, 108, 143

Taylor, J. H. \& Hulse, R. A. 1974, IAU Circular, No. 2704

van den Heuvel, E. P. J. \& Bonsema, P. T. J. 1984, A\&A, 139, L16

van den heuvel, E. P. J., van Paradijs, J. A., \& Taam, R. E. 1986, Nature, 322, 153

van Kerkwijk, M. H., Bassa, C. G., Jacoby, B. A., \& Jonker, P. G. 2005, in: Binary Radio Pulsars, ASP Conference Series vol 328, ed. F.A. Rasio \& I.H. Stairs, p. 357

Webbink, R. F., Rappaport, S. A., \& Savonije, G. J. 1983, ApJ, 270, 678

Weisberg, J. M. \& Taylor, J. H. 1981, General Relativity and Gravitation, 13, 1

Zeldovich, Ya.B. \& Guseynov, O. H. 1965, ApJ, 144, 840 